\title{
Curriculum, entre modèle rationnel et irrationalité des sociétés
}

Curricula: between rational model and irrational societies

Currículum, entre modelo racional e irracionalidad de las sociedades

Philippe Jonnaert

\section{OpenEdition}

Journals

Édition électronique

URL : https://journals.openedition.org/ries/1073

DOI : 10.4000/ries. 1073

ISSN : 2261-4265

Éditeur

France Education international

Édition imprimée

Date de publication : 1 avril 2011

Pagination : 135-145

ISBN : 978-2854205916

ISSN : $1254-4590$

\section{Référence électronique}

Philippe Jonnaert, «Curriculum, entre modèle rationnel et irrationalité des sociétés », Revue

internationale d'éducation de Sèvres [En ligne], 56 | avril 2011, mis en ligne le 01 avril 2014, consulté le

04 mai 2021. URL : http://journals.openedition.org/ries/1073 ; DOl : https://doi.org/10.4000/ries.1073

\section{(c) Tous droits réservés}




\section{Curriculum, entre modèle rationnel et irrationalité des sociétés}

\section{Philippe Jonnaert}

En 2001, Braslavsky, établissant un état des lieux du mouvement de réformes curriculaires que vivent les systèmes éducatifs à travers le monde, met en évidence l'évolution du concept même de curriculum. Le présent article s’inscrit dans ce bouillonnement contemporain des réflexions curriculaires.

\section{Pluralité d'entendements AUTOUR DE LA NOTION DE CURRICULUM}

Alors que le concept de curriculum est central dans les départements de sociologie du curriculum au sein des universités anglo-saxonnes et nord américaines, il reste un objet mal défini dans le champ franco-européen de l'éducation.

Le courant curriculaire anglo-saxon et nord américain offre à la notion de curriculum d'un système éducatif une vision qui dépasse largement celle de programme d'études. Dans la lignée des perspectives de Tyler (1950) et de Bobbitt (1918, 1924), un curriculum y est considéré comme un plan d'action pédagogique, plus large qu'un programme d'études, se situant en amont de ces programmes, en précisant les finalités, mais sans s’y limiter, spécifiant les orientations à donner aux activités d'enseignement et d'apprentissage, fournissant des indications relatives à l'évaluation, au matériel didactique, aux manuels scolaires, régissant le régime pédagogique et le régime linguistique, organisant la formation des enseignants, etc. Dans une telle vision, le curriculum dépasse largement les questions technicistes de codification de savoirs, d'écriture d'objectifs ou de formalisation de compétences dans des programmes d'études.

Ce courant curriculaire anglo-saxon et nord américain a le souci de la fonctionnalité des apprentissages et place les élèves au centre de ses préoccupations. Le rapport aux expériences de vie des apprenants y est très présent, l'influence du pragmatisme de Dewey y étant toujours perceptible. Inscrit dans une société à un moment donné de son histoire, le curriculum, tel que perçu dans cette perspective, est imprégné de dimensions culturelles, sociales et historiques : produit local, il est peu exportable. Il reste cependant ouvert au monde à travers les standards internationaux auxquels il conforme ses programmes d'études. Dans cette perspective, le curriculum devient un des moyens essentiels 
pour permettre à un système éducatif de s'adapter aux besoins en matière d'éducation et de formation d'une société à un moment donné de son histoire. Plutôt que de parler de réformes, les spécialistes des questions curriculaires, dans cette perspective, traitent plutôt d'un curriculum en développement.

Le courant curriculaire franco-européen contemporain propose une vision différente du concept de curriculum : «[...] le curriculum désigne la programmation des contenus d'enseignement tout au long de la scolarité » (Reuter, Cohen-Azria, Daunay, Delcambre, Lahanier-Reuter, 2007). Cette citation reflète bien la logique franco-européenne qui considère, aujourd'hui encore, un curriculum comme un ensemble de programmes d'études construits sur des bases disciplinaires. Le curriculum désigne alors « des contenus d'enseignement finalisés, subordonnés à des objectifs transmis méthodiquement » (Danvers, 1992). Dans la littérature franco-européenne contemporaine, curriculum et programmes d'études se superposent et traitent prioritairement de savoirs, de matières et de disciplines scolaires, de contenus d'apprentissage, de leur programmation, de leur structuration et de leur organisation. Même lorsque des auteurs s'en défendent, les savoirs scolaires restent centraux dans leur réflexion curriculaire : « Nous avons retenu le terme curriculum de préférence à ceux de programme ou de plan d'études, afin de situer la réflexion dans une perspective large sur les savoirs scolaires ${ }^{1}$, allant de leur définition à leur mise en œuvre et leur évaluation, ne réduisant pas nos interrogations aux seuls textes officiels strictement liés à telle ou telle discipline ou matière scolaire » (Audigier, Tutiaux-Guillon et Haeberli, 2008). Dans cette perspective franco-européenne, le concept même de curriculum est pratiquement superposé à celui de programme d'études. Il propose une organisation et une programmation des contenus d'apprentissage. Ce type de curriculum est centré sur la transmission de contenus, qu'ils soient définis en termes de savoirs, de compétences ou d'objectifs dans les programmes d'études.

Ces deux approches ne traitent pas du tout de la notion de curriculum dans une même logique. Le courant anglo-saxon et nord-américain place le curriculum en amont des programmes d'études qu'il oriente. Le courant francoeuropéen superpose les deux notions, utilisant souvent l'une pour l'autre. Cependant, plutôt que d'opposer ces deux perspectives, il apparaît plutôt utile de les mettre en relation et d'en rechercher les complémentarités. L'une des deux perspectives n'exclut certainement pas l'autre. La dimension programmatique des contenus d'apprentissage des curriculums francophones européens complète certainement la vision humaniste et pragmatique du curriculum anglo-saxon.

Encore faut-il préciser à quel niveau d'un curriculum cet aspect programmatique des contenus d'apprentissage peut être traité : tandis que le curriculum oriente l'action éducative dans un système éducatif, les programmes d'études définissent les contenus des apprentissages et des formations pour que

1. Non mis en évidence dans le texte original. 
les enseignants puissent mettre en application les orientations du curriculum dans leur salle de classe. Dans ce cas, le curriculum inclut nécessairement les programmes d'études, et non l'inverse, dans une relation hiérarchique. C'est à l'intérieur de ce rapport d'inclusion hiérarchique qu'un lien peut être établi entre curriculum et programmes d'études et qu'une analyse de ce rapport peut être réalisée.

\section{TROIS MOUVEMENTS CURRICULAIRES OU LA RATIONALITÉ D'UN SYSTÈmE}

Keeves décrit trois niveaux de curriculum : le curriculum prescrit (intended curriculum), le curriculum enseigné dans les salles de classe (implemented curriculum) et le curriculum assimilé par les élèves (achieved curriculum). La réalité des systèmes éducatifs et de leur curriculum est souvent très éloignée des propos, des théories et des recherches actuels sur les curriculums ${ }^{2}$. Entre les trois niveaux de curriculum décrits par Keeves, il existe bon nombre de cassures, de transformations, de médiations et de reconstructions, car la transposition des contenus du curriculum officiel jusqu'aux processus d'apprentissage par les élèves dans les salles de classe est très complexe, et certainement pas linéaire. Le cheminement d'un niveau de curriculum à l'autre n'est pas automatique, ces trois niveaux impliquant des acteurs et des lieux différents qui ne sont connectés entre eux qu'épisodiquement.

Les trois niveaux curriculaires sont présentés dans le tableau qui suit. Ils illustrent la rationalité d'un système fondé sur des niveaux enchâssés de façon logique.

Le tableau décrit globalement le travail réalisé à chacun des niveaux curriculaires $^{3}$. La plupart des recherches, travaux, documents ou réflexions curriculaires pourraient être positionnés dans un certain nombre de ces cases, mais ils n'en préciseraient guère les liens qui devraient se tisser entre chacune d'elles. Par exemple, les programmes d'études d'un système éducatif peuvent être situés dans la cellule 1.23 du tableau. Si horizontalement le cheminement d'une cellule à l'autre semble évident, verticalement le passage d'un niveau à l'autre s'avère beaucoup plus complexe. Dans ce modèle logique et hiérarchique, tout se passe comme si chacune des cellules représentait une entité homogène ouverte sur la cellule subséquente et ainsi de suite, allant sans problème du cabinet du ministre de l'éducation jusqu'à la salle de classe. Présenté ainsi, ce modèle, quoiqu'utopique, est indispensable pour comprendre le fonctionnement d'un curriculum.

2. Un important corpus théorique et empirique abonde dans la littérature sur le développement curriculaire : se référer aux revues European Educational Research Journal ; Learning and Instruction ; Journal of Curriculum Studies ; International Review of Educational Reform ; Prospects, Quartely Review of Comparative Education ; etc.

3. Adapté de Jonnaert, Charland, Cyr, Defise, Ettayebi, Furtuna, Sambote, Simbagoye et Tahirou (à paraître). 


\begin{tabular}{|c|c|c|c|c|c|}
\hline 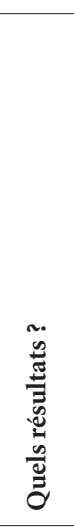 & 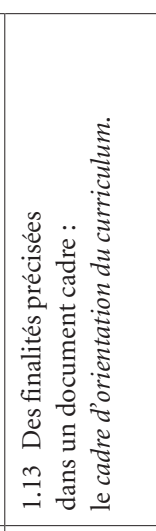 & 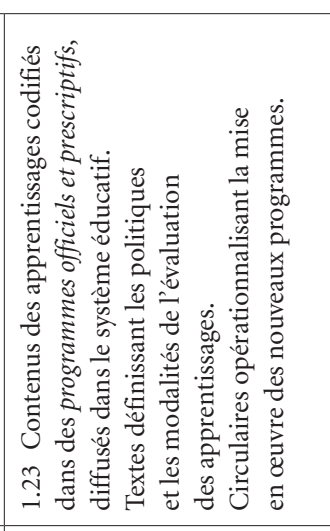 & 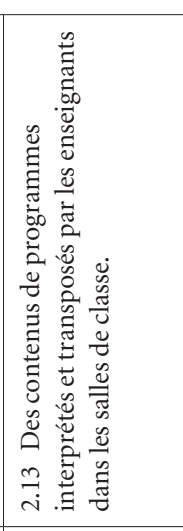 & 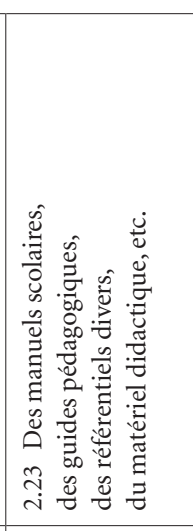 & 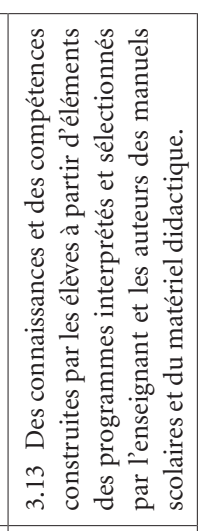 \\
\hline$\ddot{z}$ & 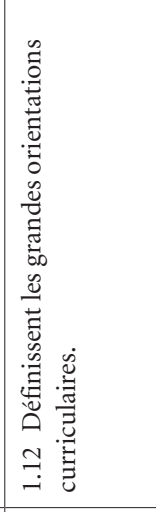 & 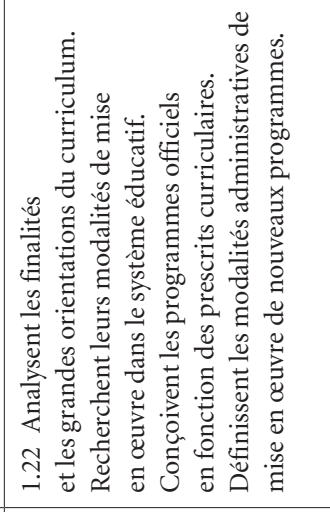 & 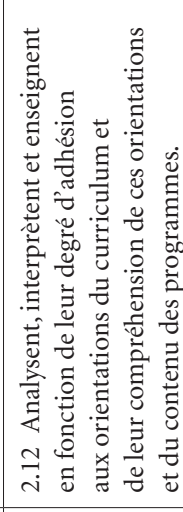 & 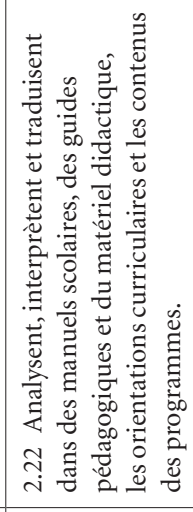 & 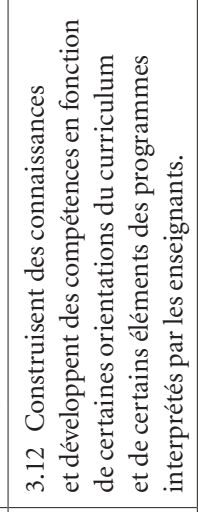 \\
\hline$\ddot{z}$ & 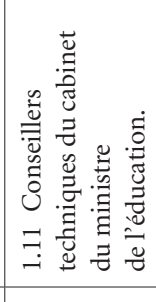 & 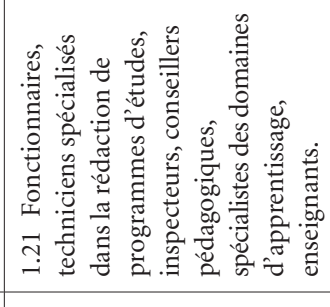 & 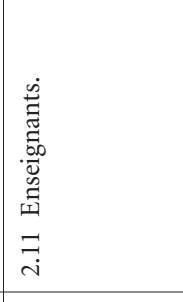 & 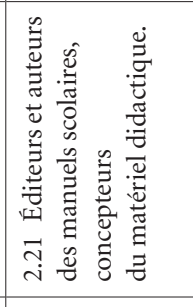 & 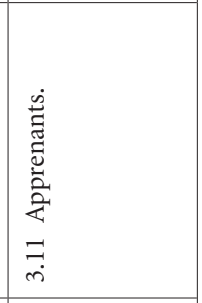 \\
\hline$\tilde{z}$ & 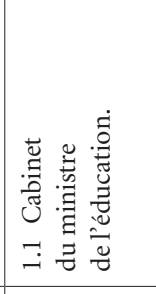 & 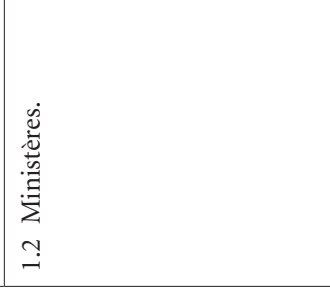 & 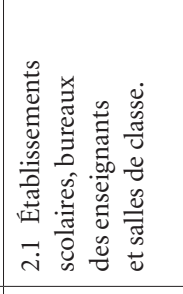 & 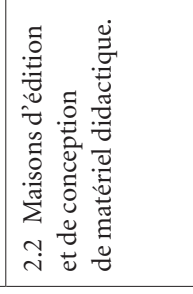 & 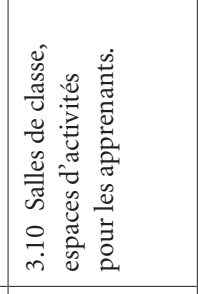 \\
\hline 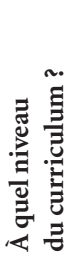 & 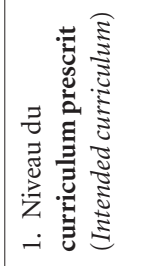 & & 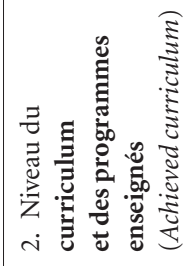 & & 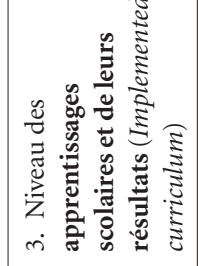 \\
\hline
\end{tabular}


Mais est-il possible de comprendre ces mouvements et ces passages d'un niveau curriculaire à un autre?

\section{HYPOTHÉTIQUE TRANSPOSITION CURRICULAIRE}

Un curriculum, dans son ensemble, articule théoriquement ces trois niveaux entre eux. Il ne s'agit pas de trois curriculums différents mais bien du cheminement d'un même curriculum, depuis sa définition officielle jusqu'aux apprentissages scolaires. L'ensemble des modifications que subit le curriculum jusqu'à son adaptation aux démarches d'enseignement et d'apprentissage dans les salles de classe constitue en fait une transposition proche de celle définie pour les didactiques des disciplines par Chevallard (1985). Cette transposition est l'illustration du dynamisme, voire de la mouvance d'un curriculum qui se déplace en se transformant et s'adaptant d'un niveau à un autre. Chacun de ces niveaux curriculaires correspond donc nécessairement à des résultats de transpositions qui ont permis leur positionnement à un moment donné du cheminement du curriculum. Mais s'agit-il encore de transposition didactique?

Chevallard cadre la transposition à l'intérieur de perspectives didactiques et disciplinaires. Il retrace certains déplacements qu'ont vécus des savoirs scolaires en mathématiques. Martinand (2001) accepte une pluralité de références pour un objet d'apprentissage. Cette transposition dite didactique est devenue un outil d'analyse intéressant pour comprendre les cheminements multiples d'un savoir à travers ses codifications dans les programmes d'études d'un curriculum donné. Codifié, le savoir écrit dans des programmes d'études ne constitue cependant qu'un texte officiel, sans plus. Son chemin est encore long avant d'aboutir à la construction de connaissances par des élèves qui, de toute façon le déforment pour l'adapter et ensuite le construire sous une forme qui leur est propre. Martinand et Chevallard conçoivent la transposition didactique comme un outil d'analyse du cheminement curriculaire de savoirs codifiés dans des programmes d'études.

Cette transposition, fût-elle didactique, en fait la transformation d'un objet de savoir dans son cheminement curriculaire. Le passage du concept de transposition didactique à celui de transposition curriculaire semble intéressant. Il est évident que la transposition didactique, pour décrypter ce voyage particulier d'un savoir jusqu'à sa codification dans des programmes d'études, voire jusqu'à son appropriation par les élèves (Jonnaert et Vanderborght, 2009), circule en des va-et-vient incessants entre les trois niveaux d'un curriculum. Auquel cas, même didactique, cette transposition est-elle autre chose qu'un outil curriculaire? Peut-on pour autant parler de transposition curriculaire? Un éventuel processus de transposition curriculaire permettrait-il cependant d'analyser autre chose que le cheminement curriculaire de savoirs codifiés dans des programmes d'études ? Par exemple cet outil curriculaire facilitera-t-il l'étude 
du cheminement d'un curriculum et de ses multiples transformations à travers les différents niveaux curriculaires?

Une telle perspective est beaucoup plus large que la simple étude du cheminement curriculaire d'un savoir codifié dans des programmes d'études. C'est la complexité d'un curriculum qui deviendrait l'objet de telles analyses à travers les mouvements d'un curriculum, ses adaptations, ses ruptures et ses incohérences. En effet, un curriculum, dans sa dimension officielle, n'est jamais injecté tel quel dans les salles de classe. Plus personne n'en disconvient. Il passe nécessairement par la médiation de l'enseignant. Cette dernière devient alors une des facettes importantes à analyser par la transposition curriculaire à travers les interprétations, les initiatives et la créativité des enseignants. Une transposition curriculaire aurait, à chacun des niveaux du curriculum, à définir des méthodologies et une véritable batterie d'outils, du focus groupe au questionnaire, de l'analyse textuelle des documents officiels à la compréhension des acquis des élèves. Se limitant au niveau de l'intended curriculum, Jonnaert, Ettayebi et Defise (2009) en suggèrent une analyse au départ de six caractéristiques et de leurs critères : (1) l'unicité du curriculum ; (2) le degré de participation des acteurs de l'éducation à son élaboration; (3) le degré d'adaptabilité du curriculum ; (4) l'univocité du curriculum ; (5) sa cohérence interne ; (6) sa cohérence externe. S'arrêtant à un seul des trois niveaux curriculaires, cette démarche est certes fort incomplète, car elle ne permet pas d'appréhender un curriculum dans son ensemble à travers ses différents niveaux. Une approche semblable à celle que ces auteurs ont définie pour le premier niveau d'un curriculum est nécessaire pour chacun des deux autres niveaux. Ce n'est qu'alors qu'une véritable transposition curriculaire opérationnelle pour l'analyse critique d'un curriculum peut devenir opérationnelle. Mais peut-on envisager un tel degré de rationalisation pour un curriculum?

\section{IRRATIONALITÉ D'UN CURRICULUM}

Suffit-il de préciser des orientations curriculaires et de les traduire dans des programmes d'études pour que tout puisse cheminer automatiquement d'un niveau de curriculum à un autre ? Suffit-il de construire des outils d'analyse pour comprendre la complexité de tout un curriculum ? Les lignes qui précèdent génèrent un ensemble de questions, chacune plus délicate que les autres, mais toutes remettent en cause le caractère automatique et linéaire du passage du curriculum officiel jusqu'aux acquis des élèves. Comment le curriculum dans sa dimension officielle franchit-il les différentes strates d'un système éducatif pour pénétrer dans les salles de classe et faire en sorte que les élèves construisent réellement des connaissances et développent effectivement des compétences relatives aux prescrits du curriculum et aux savoirs codifiés dans des programmes d'études? Comment ces savoirs codifiés peuvent-ils réellement devenir des 
objets d'apprentissage pour les élèves? Existe-t-il une réelle rétroaction depuis le curriculum implanté jusqu'au curriculum officiel ? Ce questionnement fragilise le curriculum présenté comme une structure rigide, figée, rationnelle et sans vie aucune. Un curriculum ressemble plutôt à une entité dynamique évoluant et se transformant sans cesse d'un niveau à un autre.

Pour comprendre un curriculum, le chercheur le positionne dans son environnement social et culturel avec les implications qui résultent de cette inscription dans une société donnée : «Mon métier d'ethnologue, l'étude directe ou indirecte de sociétés très différentes de la nôtre et différentes entre elles, m'a fait comprendre qu'aucune société réelle ou même possible ne peut jamais accéder à la transparence rationnelle. On ne fait pas une société à partir d'un système. Une société quelconque est d'abord faite de son passé, de ses mœurs, de ses usages : ensemble de facteurs irrationnels contre quoi les idées théoriques, qu'on prétend rationnelles, s'acharnent (...) », C. Lévi-Strauss ${ }^{4}$. Un curriculum, par son appartenance à une société donnée, est nécessairement confronté à cette irrationalité. Les systèmes rationnels comme ceux évoqués plus haut sont nécessaires, tout en n'étant pas suffisants pour analyser un curriculum. Tout curriculum se situe dans un tel paradoxe : défini par des systèmes rationnels, il évolue dans l'irrationalité d'une société donnée. Une part plus ou moins importante des curriculums analysés affiche toujours des zones d'ombre qui restent peu accessibles aux chercheurs.

Plutôt que de se présenter selon une modélisation hiérarchique et linéaire, les trois niveaux d'un curriculum devraient plutôt être en interaction constante. Des intersections devraient exister entre ces trois niveaux. Elles devraient être plus ou moins importantes en fonction des systèmes éducatifs et du type de curriculum qui s'y développe (Schiro, 2008) ${ }^{5}$. Mais ces interactions et ces intersections sont le plus souvent parasitées par des interférences multiples, rendant ce système présenté dans le tableau peu rationnel, et finalement si peu systémique lui-même. Par exemple, le retour d'informations depuis le niveau achieved curriculum vers le niveau intended curriculum est souvent réalisé par des voies parallèles, tels des syndicats d'enseignants ou d'autres groupes de pression voulant imposer telle ou telle modification au système éducatif. Le niveau implemented curriculum ne rétroagit que très rarement par les voix des élèves. Ce sont plutôt les parents qui prennent leur relais, par exemple pour contester la forme d'un bulletin scolaire ou la disparition de la dictée hebdomadaire de la grille horaire de leurs enfants. Les grandes enquêtes internationales se servent

4. Enthoven, J.-P., Burguière, A. (2009). «Un artisan, pas un maître penseur. Ce que je suis », par Claude LéviStrauss, interview au Nouvel Observateur en 1980. Nouvel Observateur Hors Série, novembre-décembre 2009, p. 30-36.

5. Schiro (2008) classe les curriculums en fonction de quatre idéologies: The scholar academic ideology; The learner centered ideology; The social efficiency ideology; The social reconstruction ideology. Les travaux des chercheurs de la CUDC démontrent qu'un curriculum est rarement univoque et qu'il se situe en général sur plusieurs de ces idéologies avec une dominante qui est l'idéologie officielle du système éducatif concerné. 
de leurs observations sur les acquis des élèves pour transmettre des informations au niveau intended curriculum, sans même que les élèves eux-mêmes en soient conscients. La rationalité d'un curriculum est sans cesse perturbée par des voix diverses, celles des partenaires de l'école et de la société civile. Ces derniers n'hésitent jamais à questionner et à remettre en cause un curriculum et ses évolutions dont ils ne perçoivent plus les fondements. La rationalité de ce modèle reste relative et les rétroactions d'un niveau à un autre sont rares et difficiles.

\section{DES NIVEAUX APPAREMMENT ÉTANCHES}

Pour comprendre le caractère souvent cloisonné des réflexions curriculaires, plusieurs travaux actuels sont décrits dans les lignes qui suivent, qui semblent souvent focaliser leurs efforts sur une seule des cellules du tableau: pour intéressants qu'ils soient, ces travaux n'abordent jamais la question curriculaire que par le petit bout de la lorgnette.

Wesselink, Dekker-Groen, Biemans, et Mulder (2010) vérifient comment douze équipes d'enseignants, impliquées dans un processus de réécriture de leurs programmes, s'approprient les orientations de leur curriculum. L'article montre comment ces enseignants, rédacteurs de programmes d'études, deviennent eux-mêmes capables de transposer de plus en plus efficacement des orientations curriculaires vers des programmes d'études. Ce travail, certes important, ne chemine pas vers les salles de classe, restant au niveau du curriculum officiel (case $1.22 \mathrm{du}$ tableau). En quoi ce prescrit curriculaire est-il pertinent pour le public ciblé par ces programmes?

Une équipe de chercheurs de la $\operatorname{CUDC}^{6}$ a formé des concepteurs nigériens ${ }^{7}$ de programmes d'études à l'intégration des orientations curriculaires prescrites dans un cadre d'orientation vers des programmes d'études. Ce travail s'achève au moment de déposer ce texte : là non plus, le cheminement jusqu'à la salle de classe n'est pas encore balisé, bien que, dans ce cas, le travail de rédaction des programmes ait été précédé par différentes activités dont une vaste enquête nationale sur les attentes des Nigériens quant au contenu de la formation de leurs enfants au cycle de base 1 .

Renaud, Chevalier, et al. (1997) évaluent l'implantation d'un programme de formation, montrant qu'un certain nombre de professeurs ont implanté ce nouveau curriculum dans leur salle de classe. Le critère retenu pour poser cette affirmation est le pourcentage de leçons extraites du guide pédagogique que ces enseignants ont appliqué dans leur cours : ce travail, qui se situe au niveau du

6. CUDC : chaire UNESCO de développement curriculaire, Université du Québec à Montréal.

7. Projet d'appui à la mise en œuvre de la réforme du curriculum de l'éducation de base 1 et du non formel au Niger, 2009/2011; partenariat entre la CUDC/UQAM et le ministère nigérien de l’Éducation. 
curriculum enseigné ne se rend pas jusqu'aux apprentissages des élèves, confondant ce travail sur des programmes d'études et des guides pédagogiques avec l'ensemble d'un curriculum.

Dolz, Jacquin et Schneuwly (2006) analysent comment un programme passe dans une salle de classe à travers l'analyse de l'objet enseigné : ils analysent le rapport de l'enseignant à l'objet d'enseignement. Mais là encore de l'objet enseigné aux résultats des apprentissages, un pas n'est pas franchi.

Tehio (2010) rassemble dans un ouvrage collectif une série d'études relatives à l'implantation de programmes d'études dans les systèmes éducatifs de cinq pays d'Afrique subsaharienne. Là encore, l'analyse curriculaire ne dépasse pas le niveau du curriculum enseigné (case 2.13 du tableau).

Tout se passe, dans bon nombre de ces travaux, comme si les élèves et leurs acquis ne relevaient pas d'une dimension curriculaire et que, par conséquent, les travaux et les recherches curriculaires pouvaient ne pas les prendre en considération. Les résultats des apprentissages scolaires n'apparaissent à travers les acquis des élèves que par les grandes évaluations nationales ou internationales. Comme cela se fait au départ de toutes les grandes enquêtes internationales, Makuwa (2010) part des données recueillies au départ des épreuves du SACMEQ ${ }^{8}$ pour évaluer les résultats des élèves dans différentes disciplines scolaires: "Le projet SACMEQ offre cette caractéristique exceptionnelle de permettre aux systèmes éducatifs participant d'évaluer scientifiquement l'évolution des résultats des élèves de $6^{\mathrm{e}}$ année du primaire en lecture et en mathématiques ». C'est au départ de ce type d'informations que des rétroactions relatives aux acquis des élèves passent du niveau de l'implemented curriculum au niveau de l'intended curriculum. Cette recherche se situe au niveau de la case 3.13 du tableau, mais finalement n'apporte rien à l'élève qui construit réellement ses connaissances dans les salles de classes. Quels que soient les résultats de ces enquêtes, il continue plus ou moins bien son cheminement d'élève. Les données recueillies au niveau micro de la salle de classe sont traitées ailleurs et n'y reviennent que filtrées à travers de multiples opérations, pour autant que l'enseignant lise les documents publiés par le SACMEQ et l'IIPE'.

Il est très difficile pour les chercheurs dont l'objet d'études est le curriculum, de trouver une cohérence entre ces travaux tant ils sont éloignés les uns des autres. Et pourtant ils peuvent chacun être positionnés dans une des cellules du tableau 1. Mais ces travaux, bien que se situant tous dans une problématique curriculaire, se cantonnent à une seule des cases du tableau 1. Ils rendent ainsi ces dernières des plus étanches, reposant la question centrale : le dynamisme d'un curriculum n'est-il qu'une utopie?

8. SACMEQ : Consortium de l'Afrique australe et orientale pour le pilotage de la qualité de l'éducation.

9. IIPE : Institut international de planification de l'éducation de l'UNESCO. L'IIPE prend une part très importante dans la réalisation des études du SACMEQ. 
L'approche du curriculum en construction par les chercheurs de la CUDC est proche de celle de Nieto, Bode, Kang et Raible (2008). Elle s'écarte d'une vision techniciste, inscrite dans un système rationnel du curriculum et centré exclusivement sur la diffusion de savoirs. Elle s'ouvre fondamentalement sur l'apprenant, son intégration dans sa communauté et son ouverture sur le monde. Cet apprenant étrangement oublié par tant de travaux curriculaires est au centre des travaux des chercheurs de la CUDC. L'approche de ces derniers intègre, dans un processus de transposition curriculaire, une ouverture aux dimensions peu rationnelles des curriculums en développement dans des sociétés aux dimensions multiples. La problématique de cette transposition des prescrits officiels d'un curriculum jusqu'aux apprentissages scolaires est au cœur des stratégies de mise à l'essai et d'implantation des curriculums dans les salles de classe. Et c'est probablement ce processus de transposition curriculaire qui se trouve au cœur du débat sur le curriculum. Les travaux et les réflexions de ces chercheurs permettent progressivement d'ouvrir la réflexion curriculaire à l'ensemble des dimensions sociétales qui sollicitent le curriculum pour la formation des personnes.

Par là, le curriculum permettra enfin aux personnes de s'adapter à la société, qui attend leur propre développement pour s'épanouir elle-même avec eux dans une société ouverte aux différences et à sa propre irrationnalité.

\section{BibLIOGRAPHIE}

AUDIGIER F., TUTIAUX-GUILLON N., HAEBERLI P. (Éd.) (2008) : Compétences et contenus. Les curriculums en questions. Bruxelles : DeBoeck.

BOBBITT J.F. (1918) : Curriculum. Boston : Houghton - Mifflin.

BOBBITT J.F. (1924). How to Make a Curriculum. Boston : Houghton - Mifflin.

BRASLAVSKY C. (2001) : Tendances mondiales et développement des curricula. Bruxelles: Association francophone d'éducation comparée (AFEC), [conférence prononcée aux journées internationales sur les politiques nationales d'éducation et de formation, (20 pages)].

CHEVALLARD Y. (1985) : La transposition didactique. Grenoble: La Pensée Sauvage.

CRAHAY M., AUDIGIER F., DOLZ J. (2006) : «Introduction. En quoi les curriculums peuvent-ils être objets d'investigation scientifique ?" In F. Audigier, M. Crahay et J. Dolz (Éd.). Curriculum, enseignement et pilotage, (7-37). Bruxelles : Deboeck.

DANVERS F. (1992) : 700 mots clés pour l'éducation. Lille: Presses universitaires de Lille.

DOLZ J., JACQUIN M., SCHNEUWLY B. (2006) : "Le curriculum enseigné en classe de français au secondaire : une approche à travers des objets enseignés ». In F. Audigier, M. Crahay et J. Dolz (Éd.). Curriculum, enseignement et pilotage, (143-164). Bruxelles : Deboeck.

JONNAERT Ph., CHARLAND P., CYR S., DEFISE R., ETTAYEBI M., FURTUNA D., SAMBOTE J.S., SIMBAGOYE A. et TAHIROU K. [à paraître] : Approche par situations. Matrice du traitement compétent de situations. Paris: UNESCO et Bureau International de l'Éducation. 
JONNAERT Ph. et VANDERBORGHT C. (2009) : Créer des conditions d'apprentissage, un cadre de référence socioconstructiviste pour la formation didactique des enseignants. Bruxelles : DeBoeck - Université, (3 édition, $1^{\text {re }}$ édition en 1998 ; traduction en portugais aux éditions Artmed).

KEEVES J. (1992) : Methodology and Measurement in International and Educational Surveys. London : Pergamon Press.

MAKUWA D.K. (2010): Acquis des élèves: des résultats contrastés, Lettre d'information de l'IIPE, XXVIII (3), 4-5.

MARTINAND J.-L. (2001) : «Pratiques de référence et problématique de la référence curriculaire ». In A. Terrisse, (Éd.), Didactique des disciplines, les références au savoir, (17-24). Bruxelles : DeBoeck - Université.

NIETO S., BODE P., KANG E. et RAIBLE J. (2008) : «Identity, Community and Diversity. Retheorizing Multicultural Curriculum for the Postmodern Era ». In F. M. Connelly, M. F. He and J. Phillion (Éd.), The Sage Handbook of Curriculum and Instruction, (179-197). London : Sage Publications.

RENAUD L., CHEVALIER S., DUFOUR R., et al. (1997). Évaluation de l'implantation d'un curriculum scolaire: pistes d'interventions pour optimiser l'adoption et l'implantation de programmes d'éducation à la santé dans les écoles, Canadian Journal of Public Health, (88)5, 351-353.

REUTER Y., COHEN-AZRIA C., DAUNAY B., DELCAMBRE I., LAHANIERREUTER D. (Éd.) (2007) : Dictionnaire des concepts fondamentaux des didactiques. Bruxelles : DeBoeck.

SCHIRO M.S. (2008) : Curriculum Theory. Conflicting Visions and Enduring. London : Sage Publications.

SCHRIVEN M. (1967) : The Methodology of Evaluation. Chicago : Rand McNally, (AERA ${ }^{10}$ Monograph Series on Curriculum Evaluation).

TEHIO V. (Éd.) (2010) : Politiques publiques en éducation, l'exemple des réformes curriculaires. Étude sur les réformes curriculaires par l'approche par compétences en Afrique. Sèvres : CIEP ${ }^{11}$.

TYLER R. W. (1950) : Basic Principles of Curriculum and Instruction. Chicago : University of Chicago Press.

WESSELINK R., DEKKER-GROEN A.M., BIEMANS H.J.A., et al. (2010) : Using an Instrument to Analyse Compétence-Based Study Programmes : Expériences of Teachers in Dutch Vocational Education and Training, Journal of Curriculum Studies, (42) 6, 813-829. 
